\title{
A MODIFIED BROYDEN-LIKE METHOD WITH 3-1 PIECEWISE NCP FUNCTION FOR GENERALIZED NONLINEAR COMPLEMENTARITY PROBLEM
}

\author{
KE SU and DAN YANG \\ College of Mathematics and Information Science \\ Hebei University \\ Baoding, 071002 \\ P. R. China \\ e-mail: yangdan7458@163.com
}

\begin{abstract}
In this paper, we propose an improved Broyden-like method for nonlinear complementarity problems by the $3-1$ piecewise NCP function. In order to reduce the amount of calculation, we introduce a parameter to avoid compute function of $F(x)$, and give the update formula of the parameter. Moreover, a new nonmonotone line search is adopted, so that only one system of equation is needed to solve and one matrix is needed to update at each iteration. In the end, under suitable conditions, the global convergent properties are proved.
\end{abstract}

\section{Introduction}

The generalized nonlinear complementarity problem (GNCP): Find a vector $x \in R^{n}$ such that

$$
F(x) \geq 0, G(x) \geq 0, F(x)^{\top} G(x)=0
$$

2010 Mathematics Subject Classification: 90C30.

Keywords and phrases: generalized nonlinear complementarity problem, 3-1 piecewise NCP function, Broyden-like method, nonmonotone.

Received September 8, 2016

(C) 2016 Scientific Advances Publishers 
where $F, G: R^{n} \rightarrow R^{n}$ is continuously differentiable. When $G(x)=x$, GNCP reduces to the classic nonlinear complementarity problem (NCP). Moreover, when $F(x)=M x+q$, with $M \in R^{n \times n}$ and $q \in R^{n}$, NCP becomes a linear complementarity problem (LCP). The generalized nonlinear complementarity problem has many important applications in engineering and economics [1,2], and its solution has been considered extensively by using special techniques in some literatures [3-8]. In the past few years, the GNCP also had been devoted a growing attention. Zhang et al. [9] reformulated the GNCP over a polyhedral as a smoothing system of equations, and then developed a smoothing Newton-type method for solving it. And, Chen and $\mathrm{Ma}$ [10] introduced a smoothing Broyden-like algorithm for the GNCP over a polyhedral cone based on the smoothing Fischer-Burmeister function. Their methods also need to introduce the smoothing parameter $\mu$ to Fischer-Burmeister function. However, $\mathrm{Pu}$ and Zhou [11], by using the piecewise NCP function, proposed a QP-free feasible method. Motivated by the above idea, we will introduce a smoothing Broyden-like algorithm for the GNCP based on 3-1 piecewise NCP function in this paper. Firstly, we transform the generalized nonlinear complementarity problem to smooth equations by 3-1 piecewise NCP function. Then, a nonmonotone line search which is based on Broyden-like algorithm be adopt to solve it. Furthermore, in Algorithm, we let $s=F(x), t=G(x)$ as the independent variable to make the algorithm run easily. And it is a solution of GNCP from any accumulation point of $x$ which is the iteration sequence generated by our algorithm.

This paper is organized as follows. In the next section, we introduce the piecewise NCP function and transform GNCP into a equivalent system of smooth equations. In Section 3, we introduce the algorithm in detail. In Section 4, we prove the algorithm to be well-defined and present the convergent properties of the presented algorithm. 


\section{Preliminaries}

Definition 2.1 ( $P_{0}$-function). $F: R^{n} \rightarrow R^{n}$ is a continuously differetiable $P_{0}$-function, i.e., for all $x, y \in R^{n}$ with $x \neq y$, there exists an index $i_{0}$ such that

$$
x_{i_{0}} \neq y_{i_{0}},\left(x_{i_{0}}-y_{i_{0}}\right)\left[F_{i_{0}}(x)-F_{i_{0}}(y)\right] \geq 0 .
$$

Definition 2.2 (NCP pair and NCP function). We call a pair $(a, b) \in R^{2}$ to be an NCP pair if $a \geq 0, b \geq 0$ and $a b=0$; a function $\Phi: R^{2} \rightarrow R$ is called an NCP function if $\Phi(a, b)=0$ if and only if $(a, b)$ is an NCP pair.

The 3-1 piecewise linear NCP function is defined as:

$$
\Phi(a, b)= \begin{cases}3 a-\frac{a^{2}}{b}, & \text { if } b \geq a>0, \text { or } 3 b>-a \geq 0, \\ 3 b-\frac{b^{2}}{a}, & \text { if } a>b>0, \text { or } 3 a>-b \geq 0, \\ 9 a+9 b, & \text { if } a \leq 0 \text { and }-a \geq 3 b, \text { or } b \leq-3 a \leq 0 .\end{cases}
$$

If $(a, b) \neq(0,0)$, then

$$
\nabla \Phi(a, b)= \begin{cases}\left(\begin{array}{c}
3-\frac{2 a}{b} \\
\frac{a^{2}}{b^{2}}
\end{array}\right), \quad \text { if } b \geq a>0, \text { or } 3 b>-a \geq 0, \\
\left(\begin{array}{c}
\frac{b^{2}}{a^{2}} \\
3-\frac{2 b}{a}
\end{array}\right), \quad \text { if } a>b>0, \text { or } 3 a>-b \geq 0, \\
\left(\begin{array}{l}
9 \\
9
\end{array}\right), & \text { if } 0 \geq a \text { and }-a \geq 3 b, \text { or } b \leq-3 a \leq 0 .\end{cases}
$$


Denote $H: R^{3 n} \rightarrow R^{3 n}$

$$
H(s, t, x)=\left(\begin{array}{c}
s-F(x) \\
t-G(x) \\
\Phi(s, t)
\end{array}\right),
$$

where $s$ approaches $F(x), t$ approaches $G(x)$ and $s=F(x), t=G(x)$ at the solution. $\Phi(s, t)$ is 3-1 piecewise linear NCP function.

Therefore, we can reform the GNCP (1.1) to the following minimization problem:

$$
\begin{aligned}
& \min \Psi(s, t, x) \\
& \Psi(s, t, x)=\|H(s, t, x)\| .
\end{aligned}
$$

\section{A modified Broyden-like Algorithm with 3-1 Piecewise NCP Function}

For solving (2.5), we need to introduce the following symbols:

$$
\left(\xi_{i}^{k}, \eta_{i}^{k}\right)=\left\{\begin{array}{lc}
(1,1) & (x, s)=(0,0), \\
\nabla \Phi(x, s) & \text { otherwise }
\end{array}\right.
$$

$i=1,2, \cdots n$, obviously, $\xi_{i}^{k}>0$ and $\eta_{i}^{k}>0$.

Compute the Jacobian matrix $V\left(s^{k}, t^{k}, x^{k}\right)$ of $H\left(s^{k}, t^{k}, x^{k}\right)$, we get

$$
V\left(s^{k}, t^{k}, x^{k}\right)=\left(\begin{array}{ccc}
I & 0 & -\nabla F\left(x^{k}\right) \\
0 & I & -\nabla G\left(x^{k}\right) \\
\operatorname{diag}\left(\xi_{i}^{k}\right) & \operatorname{diag}\left(\eta_{i}^{k}\right) & 0
\end{array}\right),
$$

where $I$ is identity matrix of $n \times n ; \nabla F\left(x^{k}\right), \nabla G\left(x^{k}\right)$ are gradient matrix of $F\left(x^{k}\right)$ and $G\left(x^{k}\right)$, respectively, $\operatorname{diag}\left(\xi_{i}^{k}\right)$ or $\operatorname{diag}\left(\eta_{i}^{k}\right)$ denotes the diagonal matrix whose $i$-th diagonal element is $\xi_{i}^{k}$ or $\eta_{i}^{k}$, respectively. 


\section{Algorithm 3.1}

Step 0. Initialization

Given initial point $\left(s^{0}, t^{0}, x^{0}\right) \in R^{3 n}, \tau \in(0,1), 0<\theta, \bar{\theta}<1$, $B_{0}=V\left(s^{0}, t^{0}, x^{0}\right), k=0$.

Step 1. If $\Psi\left(s^{k}, t^{k}, x^{k}\right)=0$, then stop. Otherwise, calculation of the search direction: Calculate $u^{k}, v^{k}$, and $d^{k}$ by solving the following linear system in $(u, v, d)$ :

$$
B_{k}\left(\begin{array}{l}
u \\
v \\
d
\end{array}\right)=\left(\begin{array}{l}
F\left(x^{k}\right)-s^{k} \\
G\left(x^{k}\right)-t^{k} \\
-\Phi\left(s^{k}, t^{k}\right)
\end{array}\right) .
$$

Step 2. Nonmonotone line search.

\section{Step 2.1. If}

$$
\begin{gathered}
\Psi\left(s^{k}+u^{k}, t^{k}+v^{k}, x^{k}+d^{k}\right) \leq \theta \Psi\left(s^{k}, t^{k}, x^{k}\right), \\
\left\|\Phi\left(s^{k}+u^{k}, t^{k}+v^{k}\right)\right\| \leq \theta \max _{0 \leq r \leq m(k)-1}\left\|\Phi^{k-r}\right\|,
\end{gathered}
$$

where $m(0)=0,0 \leq m(k) \leq \min \{m(k-1)+1, M\}, M \quad$ is a positive constant. Then let

$$
s^{k+1}=s^{k}+u^{k}, \quad t^{k+1}=t^{k}+v^{k}, \quad x^{k+1}=x^{k}+d^{k}
$$

go to Step 3, otherwise go to Step 2.2.

Step 2.2. Let

$$
s^{k+1}=s^{k}+\alpha_{k} u^{k}, \quad t^{k+1}=t^{k}+\alpha_{k} v^{k}, \quad x^{k+1}=x^{k}+\alpha_{k} d^{k},
$$

where $\alpha_{k}=\tau^{j}(0<\tau<1)$ and $j$ is the smallest non-negative integer and satisfied: 


$$
\begin{gathered}
\left\|\Phi\left(s^{k+1}, t^{k+1}\right)\right\| \leq \theta \max _{0 \leq r \leq m(k)-1}\left\|\Phi^{k-r}\right\| \\
\left\|s^{k+1}-F\left(x^{k+1}\right)+t^{k+1}-G\left(x^{k+1}\right)\right\| \leq \theta \max _{0 \leq r \leq m(k)-1}\left\|s^{k-r}-F\left(x^{k-r}\right)+t^{k-r}-G\left(x^{k-r}\right)\right\| .
\end{gathered}
$$

Step 3. Update $B_{k}$ to get $B_{k+1}$ :

$$
B_{k+1}=B_{k}+\theta_{k} \frac{\left(y^{k}-B_{k} z^{k}\right)\left(z^{k}\right)^{\top}}{\left\|z^{k}\right\|^{2}},
$$

where

$$
z^{k}=\left(\begin{array}{l}
s^{k+1} \\
t^{k+1} \\
x^{k+1}
\end{array}\right)-\left(\begin{array}{c}
s^{k} \\
t^{k} \\
x^{k}
\end{array}\right), \quad y^{k}=H\left(s^{k+1}, t^{k+1}, x^{k+1}\right)-H\left(s^{k}, t^{k}, x^{k}\right),
$$

select $\theta_{k}$ to satisfy $\left|\theta_{k}-1\right| \leq \bar{\theta}$ and matrix $B_{k+1}$ is nonsingular.

Step 4. Let $k=k+1$ and go to Step 1 .

Remark 3.1. In the above algorithm, there are two types of successive iteration: (3.5) and (3.6).

\section{Convergent Properties}

In this section, we discuss the global convergence properties of a modified Broyden-like algorithm with 3-1 piecewise NCP function.

\section{Assumption 4.1}

(a) $F, G: R^{n} \rightarrow R^{n}$ is continuously differentiable $P_{0}$-function.

(b) For initial point $\left(s^{0}, t^{0}, x^{0}\right) \in R^{3 n}$, denote the level set of $\Psi(s, t, x)$ by

$$
L\left(s^{0}, t^{0}, x^{0}\right)=\left\{(s, t, x) \in R^{3 n} \mid \Psi(s, t, x) \leq \Psi\left(s^{0}, t^{0}, x^{0}\right)\right\} .
$$


Defined by (4.1), the level set of $\Psi(s, t, x)$ is bounded.

Remark 4.1. $F(x), G(x)$ are $P_{0}$-function, then $\nabla F(x), \nabla G(x)$ is positive semidefinite.

Lemma 4.1. If $H\left(s^{0}, t^{0}, x^{0}\right) \neq 0$, then $B_{0}=V\left(s^{0}, t^{0}, x^{0}\right)$ is nonsingular.

Proof. Assume $H\left(s^{0}, t^{0}, x^{0}\right) \neq 0$. If $V\left(s^{0}, t^{0}, x^{0}\right) \cdot(u, v, d)^{\top}=0$ for some $(u, v, d) \in R^{3 n}$, where $u=\left(u_{1} \cdots u_{n}\right)^{\top}, v=\left(v_{1} \cdots v_{n}\right)^{\top}, d=\left(d_{1} \cdots d_{n}\right)^{\top}$, then

$$
\begin{gathered}
I u-\nabla F\left(x^{0}\right) d=0, \\
I v-\nabla G\left(x^{0}\right) d=0, \\
\operatorname{diag}\left(\xi^{0}\right) u+\operatorname{diag}\left(\eta^{0}\right) v=0 .
\end{gathered}
$$

From the definitions of $\xi_{i}^{0}$ and $\eta_{i}^{0}$, we know that $\xi_{i}^{0}>0$ and $\eta_{i}^{0}>0$ for all $i, i=1 \cdots n$. So, $\operatorname{diag}\left(\eta^{0}\right)$ is nonsingular. By (4.4), we have

$$
v=-\left(\operatorname{diag}\left(\eta^{0}\right)\right)^{-1} \operatorname{diag}\left(\xi^{0}\right) u .
$$

Substitute $v$ in (4.5) by (4.3), we have

$$
d=-\left(\nabla G\left(x^{0}\right)\right)^{-1}\left(\operatorname{diag}\left(\eta^{0}\right)\right)^{-1} \operatorname{diag}\left(\xi^{0}\right) u .
$$

Substitute $d$ in (4.6) by (4.2), and multiplying by $u^{\top}$, we have

$$
u^{\top} I u+u^{\top} \nabla F\left(x^{0}\right)\left(\nabla G\left(x^{0}\right)\right)^{-1}\left(\operatorname{diag}\left(\eta^{0}\right)\right)^{-1} \operatorname{diag}\left(\xi^{0}\right) u=0 .
$$

The fact that $F(x), G(x)$ is the $P_{0}$-function, so all principal minor determinant of the $\nabla F(x), \nabla G(x)$ is non-negative, that is to say, $\nabla F\left(x^{0}\right),\left(\nabla G\left(x^{0}\right)\right)^{-1} \quad$ is positive semidefinite. And matrix $\left(\operatorname{diag}\left(\eta^{0}\right)\right)^{-1} \operatorname{diag}\left(\xi^{0}\right)$ is positive definite. So $u=0$. By (4.4) and (4.5), we know $v=0, d=0$. Hence, $B_{0}$ is nonsingular. 
Lemma 4.2. Suppose the Assumption 4.1 holds, $\Phi\left(s^{k}, t^{k}\right) \rightarrow 0$, as $k \rightarrow \infty$.

Proof. In view of convenience, if for all sufficiently large $k$ (3.4), (3.8) holds, define $\left\|\Phi^{l(k)}\right\|=\max _{0 \leq r \leq m(k)-1}\left\|\Phi^{k-r}\right\|$, where $k-m(k)+1 \leq l(k) \leq k$.

Since $m(k+1) \leq m(k)+1$, then

$$
\begin{aligned}
\left\|\Phi^{l(k+1)}\right\| & =\max _{0 \leq r \leq m(k+1)-1}\left\|\Phi^{k+1-r}\right\| \\
& \leq \max _{0 \leq r \leq m(k)}\left\|\Phi^{k+1-r}\right\| \\
& =\max \left\{\left\|\Phi^{l(k)}\right\|,\left\|\Phi^{k+1}\right\|\right\} \\
& =\left\|\Phi^{l(k)}\right\| .
\end{aligned}
$$

So, $\left\|\Phi^{l(k)}\right\|$ is monotone decreasing, which implies that the $\left\{\left\|\Phi^{l(k)}\right\|\right\}$ converges. It follows from (3.4), (3.8) that $\left\|\Phi^{l(k)}\right\| \leq \theta\left\|\Phi^{l(l(k)-1)}\right\|$.

Since $\theta \in(0,1)$, therefore $\left\{\left\|\Phi^{l(k)}\right\|\right\} \rightarrow 0(k \rightarrow \infty)$.

Therefore $\left\|\Phi^{k+1}\right\| \leq \theta\left\|\Phi^{l(k)}\right\| \rightarrow 0(k \rightarrow \infty)$ holds by the Algorithm 3.1. That is, $\lim _{k \rightarrow \infty}\left\|\Phi^{k}\right\|=0$.

Lemma 4.3. Suppose the Assumption 4.1 holds, $\left[s^{k}-F\left(x^{k}\right)+t^{k}-\right.$ $\left.G\left(x^{k}\right)\right] \rightarrow 0$, as $k \rightarrow \infty$.

The proof is similar to Lemma 4.2.

Lemma 4.4. Suppose the Assumption 4.1 holds, $u^{k} \rightarrow 0, v^{k} \rightarrow 0$, $d^{k} \rightarrow 0, H\left(s^{k}, t^{k}, x^{k}\right) \rightarrow 0$, as $k \rightarrow \infty$. 
Proof. In Algorithm 3.1, there are two types of successive iteration: (3.5) and (3.6).

For iteration (3.5), it satisfied (3.3). So, $\Psi\left(s^{k}, t^{k}, x^{k}\right)$ is monotone decreasing. And $\Psi(s, t, x) \geq 0$. Hence, $\Psi\left(s^{k}, t^{k}, x^{k}\right) \rightarrow 0$, as $k \rightarrow \infty$. Namely, $H\left(s^{k}, t^{k}, x^{k}\right) \rightarrow 0$, as $k \rightarrow \infty$.

For iteration (3.6), it satisfied (3.7) and (3.8). By Lemmas 4.2 and 4.3, we know $\Phi\left(s^{k}, t^{k}\right) \rightarrow 0,\left[s^{k}-F\left(x^{k}\right)+t^{k}-G\left(x^{k}\right)\right] \rightarrow 0$, as $k \rightarrow \infty$. So $H\left(s^{k}, t^{k}, x^{k}\right) \rightarrow 0$, as $k \rightarrow \infty$.

Therefore,

$$
B_{k}\left(\begin{array}{l}
u^{k} \\
v^{k} \\
d^{k}
\end{array}\right)=\left(\begin{array}{l}
F\left(x^{k}\right)-s^{k} \\
G\left(x^{k}\right)-t^{k} \\
-\Phi\left(s^{k}, t^{k}\right)
\end{array}\right) \rightarrow 0, \text { as } k \rightarrow \infty .
$$

In Algorithm 3.1, $B_{k}$ is nonsingular. So, $u^{k} \rightarrow 0, v^{k} \rightarrow 0, d^{k} \rightarrow 0$, as $k \rightarrow \infty$.

Theorem 4.1. Suppose the Assumption 4.1 holds, Equation (3.2) is solvable, Algorithm 3.1 is well-defined.

Proof. We know $B_{0}$ is nonsingular by Lemma 4.1. And produced $B_{k}$ of Algorithm 3.1 is nonsingular. So the Equation (3.2) has one and only one solution. Otherwise, we know $H\left(s^{k}, t^{k}, x^{k}\right) \rightarrow 0$, as $k \rightarrow \infty$ by Lemma 4.4. So, Algorithm 3.1 is well-defined.

\section{Acknowledgements}

This work is supported by the National Natural Science Foundation of China (No. 11101115), the Natural Science Foundation of Hebei Province (No. A2014201033), the Key Research Foundation of Education Bureau of Hebei Province (No. ZD2015069), and the Post-Graduate's Innovation Fund Project of Hebei University (No. X2016060). 


\section{References}

[1] M. C. Ferris and J. S. Pang, Engineering and economic applications of complementarity problems, SIAM Review 39(4) (1997), 669-713.

[2] Vipin Gopal and Lorenz T. Biegler, Smoothing methods for complementarity problems in process engineering, American Institute of Chemical Engineers 45(7) (1999), 1535-1547.

[3] C. Kanzow and M. Fukushima, Equivalence of the generalized complementarity problem to differentiable unconstrained minimization, Journal of Optimization Theory and Applications 90(3) (1996), 581-603.

[4] Houyuan Jiang, Masao Fukushima, Liqun Qi and Defeng Sun, A trust region method for solving generalized complementarity problems, SIAM Journal on Optimization 8(1) (1998), 18.

[5] X. Chen, D. Sun and L. Qi, Global and superlinear convergence of the smoothing Newton method and its application to general box constrained variational inequalities, Mathematics of Computation 67(222) (1998), 519-540.

[6] Roberto Andreani, Ana Friedlander and Sandra A. Santos, On the resolution of the generalized nonlinear complementarity problem, SIAM Journal on Optimization 12(2) (2001), 303-321.

[7] Yiju Wang, Fengming Ma and Jianzhong Zhang, A nonsmooth L-M method for solving the generalized nonlinear complementarity problem over a polyhedral cone, Applied Mathematics and Optimization 52(1) (2005), 73-92.

[8] Yiju Wang, Xinzhen Zhang and Liqun Qi, Unconstrained optimization reformulation of the generalized nonlinear complementarity problem and related method, Optimization 54(6) (2005), 563-577.

[9] Xinzhen Zhang, Hefeng Jiang and Yiju Wang, A smoothing Newton-type method for generalized nonlinear complementarity problem, Journal of Computational and Applied Mathematics 212(1) (2008), 75-85.

[10] Bilian Chen and Changfeng Ma, Superlinear/quadratic smoothing Broyden-like method for the generalized nonlinear complementarity problem, Nonlinear Analysis. Real World Applications 12(2) (2011), 1250-1263.

[11] Dingguo $\mathrm{Pu}$ and Yan Zhou, Piecewise linear NCP function for QP free feasible method, Applied Mathematics: A Journal of Chinese Universities 21(3) (2006), 289-301. 